\title{
Vehicle state estimation based on PSO-RBF neural network
}

Yingjie Liu*

School of Mechanical-Electronic and Vehicle Engineering,

Weifang University,

Weifang 261061, China

Email: ufoliuyingjie@163.com

*Corresponding author

\section{Qiuyun Sun}

School of Management and Information,

Shandong Transport Vocational College,

Weifang 261206, China

Email: zhuomazexi1015@126.com

\section{Dawei Cui}

School of Mechanical-Electronic and Vehicle Engineering,

Weifang University,

Weifang 261061, China

Email:wfxycdw@163.com

\begin{abstract}
In the last few years, many closed-loop control systems have been introduced in the automotive field to increase the level of safety and driving automation. For the integration of such systems, it is critical to estimate motion states and parameters of the vehicle that are not exactly known or that change over time. In order to estimate the motion states and parameters, a method based on PSO-RBF neural network is presented to solve problem of vehicle state estimation in vehicle handling dynamics. The basic idea behind the work was to identify several key parameters which affected the performance of vehicle by experimental data. Then the test data was input to the simulation model for network training and verification. The results show that the method can estimate vehicle state successfully with small absolute error of side slip angle in vehicle handling dynamics. Results are included to demonstrate the effectiveness of the estimation approach and its potential benefit towards the implementation of adaptive driving assistance systems or to automatically adjust the parameters of onboard controllers as well as the effectiveness of the proposed scheme in the estimation of states and unknown inputs.
\end{abstract}

Keywords: active safety; state estimation; PSO-RBF; neural network.

Reference to this paper should be made as follows: Liu, Y., Sun, Q. and Cui, D. (2019) 'Vehicle state estimation based on PSO-RBF neural network', Int. J. Vehicle Safety, Vol. 11, No. 1, pp.93-106. 


\section{Introduction}

The safety of vehicular traffic is the eternal theme of the development. Along with rapid increase in road traffic accidents, particularly viciously high incidence of traffic accidents, traffic safety problems have become increasingly prominent. Automotive engineering and research community along with car constructors are looking for solutions to reduce and prevent accidents, and as a consequence to improve the safety during vehicle driving.

Vehicle status estimation has an important role in improving critical safety systems. With the increase in the accuracy of wheel speed sensors and yaw rate sensors, as well as the reliability of these sensors, state estimation is an effective way to improve the control system (such as ESP performance). Therefore, it is the key problem to study the state information of automobile dynamic control system accurately and in real time.

The performance of driving safety systems may be improved if the unknown parameters of the underlying vehicle model can be measured and updated. Weight of the vehicle, road adhesion, drag coefficient and tyre cornering stiffness are examples of unknown parameters (Bektache et al., 2014; Funke and Gerdes, 2016; Rajaram and Subramanian, 2016; Chen et al., 2013a; Chen and Lee, 2011).

The vehicle state estimation problem has been studied in the literatures. A brief review is presented in this section.

Giulio et al. (2017) presented a model-based observer to estimate motion states and parameters of the vehicle that change over time with the purpose of increasing level of safety and driving automation.

Fei et al. (2015) introduced a novel nonlinear observe based on a vehicle dynamics model and a simplified Pacejka tyre model in order to provide estimation of longitudinal and lateral vehicle velocities and the tyre-road friction coefficient for vehicle safety control systems, specifically anti-lock braking control.

Pence et al. (2014) presented a recursive estimation/detection technique for reducedorder state space systems by building a recursive state and parameter estimator on the framework of polynomial chaos theory and maximum likelihood estimation.

Rath et al. (2015) developed a combination of nonlinear Lipschitz observer and modified super-twisting algorithm (STA) observer to estimate these unknown inputs and states simultaneously and also under Lipschitz conditions for the nonlinear functions, the convergence of the estimation error was established.

$\mathrm{Xu}$ et al. (2016) proposed a fusion methodology for integrating a single-frequency double-antenna Global Positioning System (DA-GPS) with other low-cost in-vehicle sensors to achieve reliable estimation of both vehicle sideslip and yaw angles.

Hui et al. (2016) developed an alternative approach which estimated the side slip angle with the measurements of relatively cheap sensors to monitor the lateral stability or improve the stability by using the feedback control.

Bangji et al. (2016) presented a novel observer design for simultaneous estimation of vehicle steering angle and sideslip angle so that the estimation of sideslip angle does not require the measurement of steering angle and the estimate of steering angle can also be used for other purposes.

Mourad et al. (2016) presented a nonlinear adaptive observer for the estimation of the wheel stiffness and radius which will be used for controller synthesis and supervision for vehicle applications. The proposed adaptive observer uses the angular velocity as an input of the system, whereas the angular position and the vehicle velocity are considered 
as a measured state vector. The adaptive observer is designed based on a nonlinear model of the vehicle quarter model and the adaptive law of the parameters is derived using Lyapunov analysis.

Ehsan et al. (2016) developed a longitudinal vehicle velocity estimator robust to road conditions by employing a tyre model at each corner and presented a new Kalman-based observer by considering tyre nonlinearities with a minimum number of required tyre parameters and the road condition as uncertainty.

Freeman et al. (2016) investigated the performance of two control algorithms, sliding control and linear quadratic control for use in an autonomous run-off-road vehicle recovery system for vehicle state estimation and control.

The above methods can solve the vehicle state estimation problem effectively but have some drawbacks such as difficulty in numbers of estimated parameters, dependency on precision of model of the vehicle.

This paper aims to present an algorithm based on PSO-RBF neural network theory for vehicle state estimation problem. The method is used to estimate motion states and parameters of the vehicle that are not exactly known and that change over time. The rest of the paper is organised as follows: Section 2 presents the 7-DOF vehicle model. Section 3 presents the PSO-RBF neural network for vehicle state estimation problem. Section 4 illustrates the numerical simulation and discussion. Finally, Section 5 summarises the conclusions and suggests future research directions.

\section{Nonlinear vehicle dynamics model}

\subsection{Model of vehicle movement}

For simplicity, the movement of the vehicle is described by a 7-DOF vehicle model depicted in Figure 1. The vehicle model has the following longitudinal, lateral, yaw and turning degrees of freedom.

Figure 1 7-DOF vehicle model

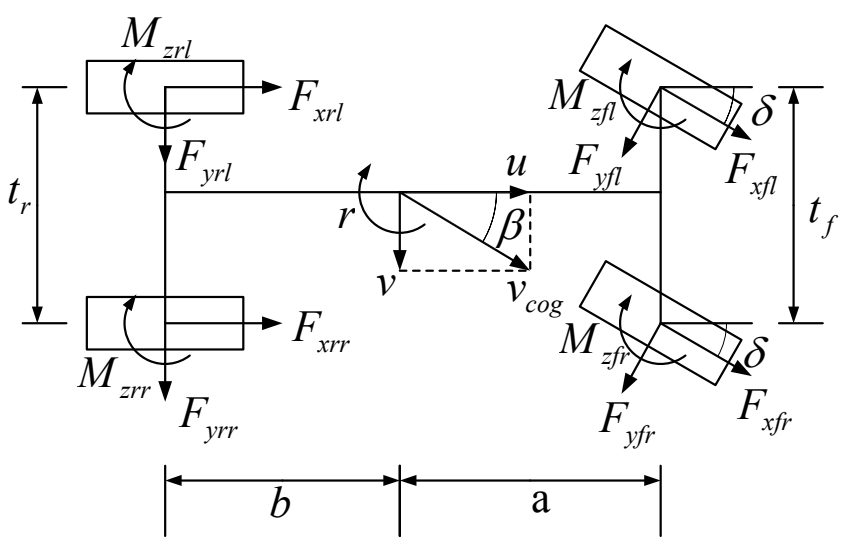


The differential equation of the movement of the vehicle is:

$$
\left\{\begin{aligned}
\dot{u} & =a_{x}+v r \\
a_{x} & =\left(F_{x f l} \cos \delta+F_{x f r} \cos \delta+F_{x r l}+F_{x r r}-F_{y f l} \sin \delta-F_{y f r} \sin \delta\right) / m \\
\dot{v} & =a_{y}-u r \\
a_{y}= & \left(F_{x f l} \sin \delta+F_{x f r} \sin \delta+F_{y f l} \cos \delta+F_{y f r} \cos \delta+F_{y r l}+F_{y r r}\right) / m \\
\Gamma & =\frac{t_{f}}{2} F_{x f l}^{\prime}-\frac{t_{f}}{2} F_{x f r}^{\prime}+\frac{t_{r}}{2} F_{x r l}-\frac{t_{r}}{2} F_{x r r}+a F_{y f l}^{\prime}+a F_{y f r}^{\prime} \\
& -b F_{y r l}-b F_{y r r}+M_{z f l}+M_{z f r}+M_{z r l}+M_{z r r} \\
\dot{r} & =\frac{\Gamma}{I_{z}}
\end{aligned}\right.
$$

where

$$
\begin{aligned}
& F_{x i j}^{\prime}=F_{x i j} \cos \delta-F_{y i j} \sin \delta \\
& F_{y i j}^{\prime}=F_{y i j} \cos \delta-F_{x i j} \sin \delta
\end{aligned}
$$

It is assumed that the moment of inertia resistance and the role of air lift are ignored, and then the vertical load of each tyre can be described as:

$$
\left\{\begin{array}{l}
F_{z f l, z f r}=\left(\frac{m g}{2} \pm m a_{y} \frac{h}{t_{f}}\right) \frac{b}{l}-\frac{1}{2} m a_{x} \frac{h}{l} \\
F_{z r l, z r r}=\left(\frac{m g}{2} \pm m a_{y} \frac{h}{t_{r}}\right) \frac{a}{l}+\frac{1}{2} m a_{x} \frac{h}{l}
\end{array}\right.
$$

The slip angle of each tyre can be obtained by the following formula (Wenzel et al., 2007):

$$
\left\{\begin{array}{r}
\alpha_{f l, f r}=\delta-\operatorname{arctg} \frac{v+a r}{u \pm \frac{t_{f}}{2} r} \\
\alpha_{r l, r r}=-\operatorname{arctg} \frac{v-b r}{u \pm \frac{t_{r}}{2} r}
\end{array}\right.
$$

The side slip angle of the vehicle is:

$$
\beta=\operatorname{arctg} \frac{v}{u}
$$

The slip ratio of each tyre is:

$$
s_{i j}=\frac{r_{e} \omega_{i j}-u_{w i j}}{u_{w i j}}
$$


where the wheel centre speed $u_{w i j}$ of each wheel can be obtained by the following formula (Kiencke and Nielsen, 2000):

$$
\left\{\begin{array}{l}
u_{w f l, w f r}=v_{\operatorname{cog}} \pm r\left(\frac{t_{f}}{2} \pm a \beta\right) \\
u_{w r l, w r r}=v_{\operatorname{cog}} \pm r\left(\frac{t_{r}}{2} \mp b \beta\right)
\end{array}\right.
$$

where $m$ is the vehicle mass, $v_{c o g}$ is the centroid speed, $I_{z}$ is the moment of inertia around the $\mathrm{z}$ axis, $v$ and $u$ are the lateral and longitudinal speed, $\Gamma$ is the yaw moment around the $\mathrm{z}$ axis, $\alpha_{x}$ and $\alpha_{y}$ are the longitudinal and lateral acceleration, $r$ is the yaw rate of the vehicle, $\beta$ is the side slip angle, $F_{x i j}$ are the longitudinal forces generated on tyres, $F_{v i j}$ are the lateral forces generated in the tyres, $F_{z i j}$ is the vertical load of each wheel, $t_{f}$ and $t_{r}$ are the front and rear tracks, $\delta$ is the front steering angle, $h$ is the centroid height, $\alpha_{i j}$ is the slip angle of each tyre, $s_{i j}$ is the slip ratio of each tyre, $M_{z i j}$ is the aligning torque of each wheel, $a$ and $b$ are the distances of front and rear axles from the centre of gravity, $L=a+b$ is the wheelbase, $r_{e}$ is the rolling radius of wheel, $\varpi_{i j}$ is the angular velocity of each wheel, $u_{w i j}$ is the centre speed of each wheel.

\subsection{Tyre model}

In this paper the Pacejka tyre model is used to simulate the movement of the vehicle. For the model the tyre vertical load, side angle and slip rate are set as input variables. And the lateral force, longitudinal force and aligning torque can be calculated by equation (7) (Bakker and Pacejka, 1989).

$$
\left\{\begin{array}{l}
y(x)=D \sin (\operatorname{Carctg}(B x-E(B x-\operatorname{arctg} B x)) \\
Y(X)=y(x)+s_{v} \\
x=X+s_{h}
\end{array}\right.
$$

where the output $Y$ represents the longitudinal force $F_{x}$ and the lateral force $F_{y}$ as well as the aligning torque $M_{z}$; the input $X$ represents the slip ratio $S$ and the slip angle $\alpha$; $B=0.237, C=1.65, D=3610.5, E=0.707, s_{v}=40.379, s_{h}=0.0473$.

\subsection{Road model}

In this paper the double lane change test road is used for road input. The double lane change test road reflects the vehicle avoiding obstacles or overtaking in high-speed when operating the steering performance.

The double lane change test road is shown in Figure 2. In Figure 2, $s_{0}=s_{1}=s_{2}=s_{4}=2 u, s_{3}=u, s_{5}=5 u, s_{6}=3 u$. $B$ is the lane change distance, $B=3.5 \mathrm{~m}$. $B_{1}, B_{2}$ and $B_{3}$ are the distances between the stakes, $B_{1}=1.1 L+0.25=2.12 \mathrm{~m}$, $B_{2}=1.2 L+0.25=2.29 \mathrm{~m}, B_{3}=1.3 L+0.25=2.46 \mathrm{~m}$, where $L$ is the width of the vehicle, $L=1.7 \mathrm{~m}$. 
Figure 2 Double lane change test road (• stands for stake)

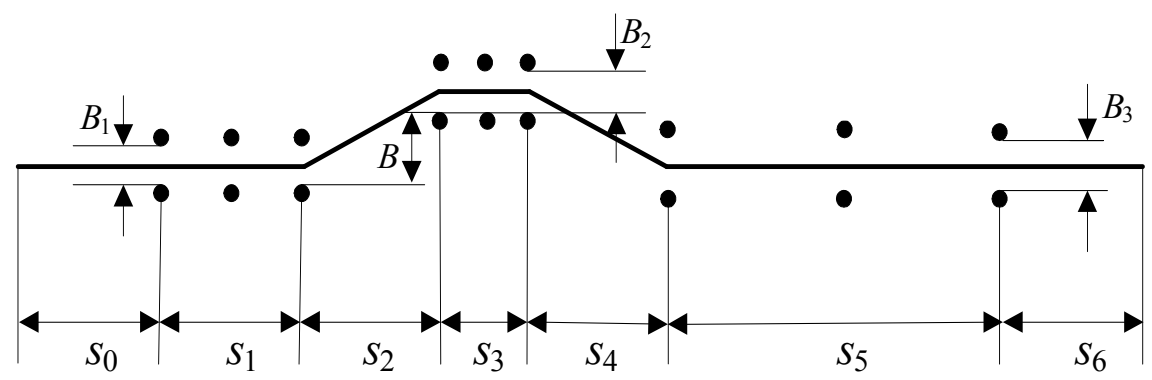

In realistic driving process, drivers' ideal target trajectory should be low-level continuous smooth curve shown in Figure 3. And also, according to ISO/TR3888-2004, the trajectory of the vehicle traveling along the pylon course slalom test road should be described as a curve of order three with continuous first-order derivative transformed with cubic splines fitting:

$$
f(x)=\left\{\begin{array}{cc}
0 & x \in s_{1} \\
e_{0}+e_{1} x+e_{2} x^{2}+e_{3} x^{3} & x \in s_{2} \\
B & x \in s_{3} \\
e_{0}^{\prime}+e_{1}^{\prime} x+e_{2}^{\prime} x^{2}+e_{3}^{\prime} x^{3} & x \in s_{4} \\
0 & x \in s_{5}
\end{array}\right.
$$

where

$$
\begin{aligned}
& a_{0}=s_{1} ; a_{1}=s_{1}+s_{2} ; \\
& a_{2}=s_{1}+s_{2}+s_{3} ; a_{3}=s_{1}+s_{2}+s_{3}+s_{4} ; \\
& d=a_{1}-a_{0} ; d^{\prime}=a_{2}-a_{3} ; \\
& e_{0}=a_{0}^{2}\left(3 a_{1}-a_{0}\right) B / d^{3} ; e_{1}=-6 a_{0} a_{1} B / d^{3} ; \\
& e_{2}=3\left(a_{0}+a_{1}\right) B / d^{3} ; e_{3}=-2 B / d^{3} ; \\
& e_{0}{ }^{\prime}=a_{3}^{2}\left(3 a_{2}-a_{3}\right) B / d^{\prime 3} ; e_{1}{ }^{\prime}=-6 a_{3} a_{2} B / d^{\prime 3} ; \\
& e_{2}{ }^{\prime}=3\left(a_{3}+a_{2}\right) B / d^{\prime 3} ; e_{3}{ }^{\prime}=-2 B / d^{\prime 3} .
\end{aligned}
$$

From equation (8) it is easy to obtain the relationship between $y$, i.e. $f(x)$ and $x$ by substituting $t$ with $x / u$.

$$
f(t)=\left\{\begin{array}{cc}
0 & t \in t_{1} \\
g_{0}+g_{1} t+g_{2} t^{2}+g_{3} t^{3} & t \in t_{2} \\
b & t \in t_{3} \\
h_{0}+h_{1} t+h_{2} t^{2}+h_{3} t^{3} & t \in t_{4} \\
0 & t \in t_{5}
\end{array}\right.
$$

where $g_{0}=e_{0} ; g_{i}=e_{i} u^{j}(j=1,2,3) ; h_{0}=e_{0}^{\prime} ; h_{i}=e_{i}^{\prime} u^{j}(j=1,2,3)$. 
Figure 3 Fitted double lane change test road

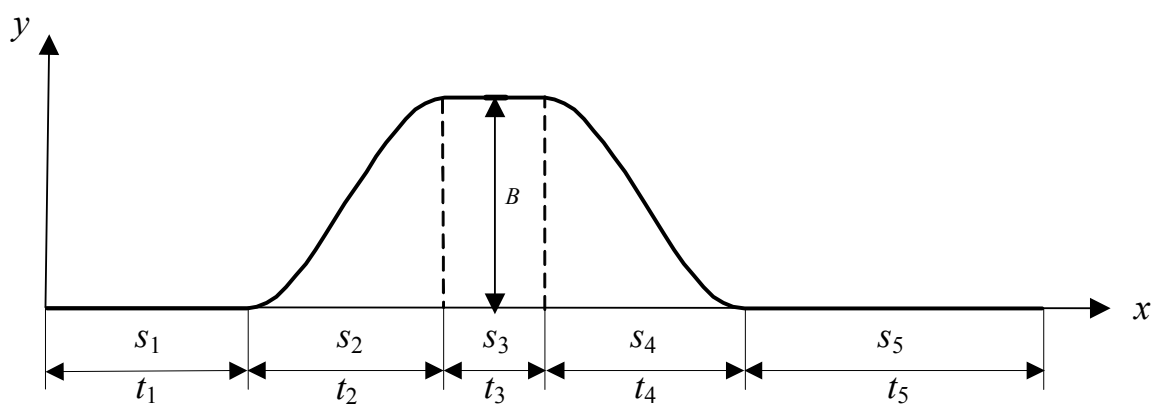

\section{PSO-RBF neural network}

RBF neural network is a type of forward neural network. The first layer of the network is the input layer, composed of source node; the second layer is the hidden layer and the number of the hidden layer nodes depends on the specific problem; the third layer is the output layer responding to the input model (Lin et al., 2013; Jeon and Beak, 2011).

The RBF used in the RBF neural network is the Gauss function. Then the output of the network is:

$$
\begin{aligned}
y_{i} & =\sum_{i=1}^{n} \varpi_{i j} \exp \left(-\frac{1}{2 \sigma^{2}}\left\|\mathbf{x}_{p}-\mathbf{c}_{i}\right\|^{2}\right) \\
i & =1,2, \cdots, h \quad j=1,2, \cdots, n \\
\mathbf{x}_{p} & =\left(x_{p 1}, x_{p 2}, \cdots, x_{p m}\right)
\end{aligned}
$$

where $\mathbf{x}_{p}$ is the $p$-th input sample, $p=1,2, \ldots, P$, and $P$ is the total number of samples; $\mathbf{c}_{i}$ is the centre of the hidden layer node; $\varpi_{i j}$ is the connection weight of the hidden layer to the output layer, and $i$ is the number of the hidden layer nodes in the neural network; $y_{i}$ is the actual output of the $j$-th output node of the network corresponding to the input sample; $\sigma$ is the variance of the Gauss function.

It is assumed that $d$ is the expected output value of the sample, then the variance of the basis function can be expressed as (Chen et al., 2013b; Tan et al., 2011):

$$
\sigma=\frac{1}{P} \sum_{i=1}^{m}\left\|d_{i}-y_{i} c_{i}\right\|^{2}
$$

The PO-RBF neural network requires training parameters such as the clustering centre of the radial basis function, the maximum distance of the centre of the radial basis and the weight between hidden layer and the output layer in the RBF neural network. The prediction is carried out in three phases. In the first stage, the centre of the RBF neural network is calculated by using the particle swarm optimisation algorithm. When the clustering centre of the radial basis function is calculated, the clustering centre is adjusted by the particle swarm optimisation algorithm, and the optimal clustering centre is the 
final clustering centre of the RBF neural network. The second stage, the K-means clustering method, is used to calculate the width of the RBF neural network. In the third stage, the network is trained and the iterative calculation is repeated.

During each iteration, the particles update their own speed and positions through individual extremes and global extremes:

$$
\begin{aligned}
& v_{i d}^{(m+1)}=\varpi v_{i d}^{(m)}+c_{1} r_{1}\left(P_{i d}^{(m)}-X_{i d}^{(m)}\right)+c_{2} r_{2}\left(P_{g d}^{(m)}-X_{i d}^{(m)}\right) \\
& X_{i d}^{(m+1)}=X_{i d}^{(m)}+v_{i d}^{(m+1)} d=1,2, \cdots, D ; i=1,2, \cdots, n
\end{aligned}
$$

where $i$ is the $i$-th particle; $\varpi$ is the inertia weight; $m$ is the current number of iterations; $d$ represents the $d$-th-dimension of the particle $i ; X_{i d}$ is the position of the $i$-th particle; $P_{i d}$ is the individual optimal position of the $i$-th particle; $P_{g d}$ is the position of the optimal fitness value for all particles; $v_{i d}$ is the velocity of the particle $i ; c_{1}, c_{2}$ is the acceleration factor; $r_{1}, r_{2}$ is the random number distributing between $[0,1]$.

In the iterative process of the algorithm, the larger inertia weight $\varpi$ is favourable to the global search and the smaller $\varpi$ is more conducive to the local search. The linear recursive inertia weight is used in the calculation:

$$
\varpi(k)=\varpi_{\text {start }}-\left(\varpi_{\text {start }}-\varpi_{\text {end }}\right)\left(T_{\max }-k\right) / T_{\max }
$$

where $\varpi_{\text {start }}$ is the initial inertia weight; $\varpi_{\text {end }}$ is the inertia weight iterating to the maximum value of times; $T_{\max }$ is the maximum number of iterations (Zhang et al., 2016).

The specific PSO-RBF algorithm flow is shown in Figure 4.

Figure 4 PSO-RBF algorithm flow for vehicle state estimation

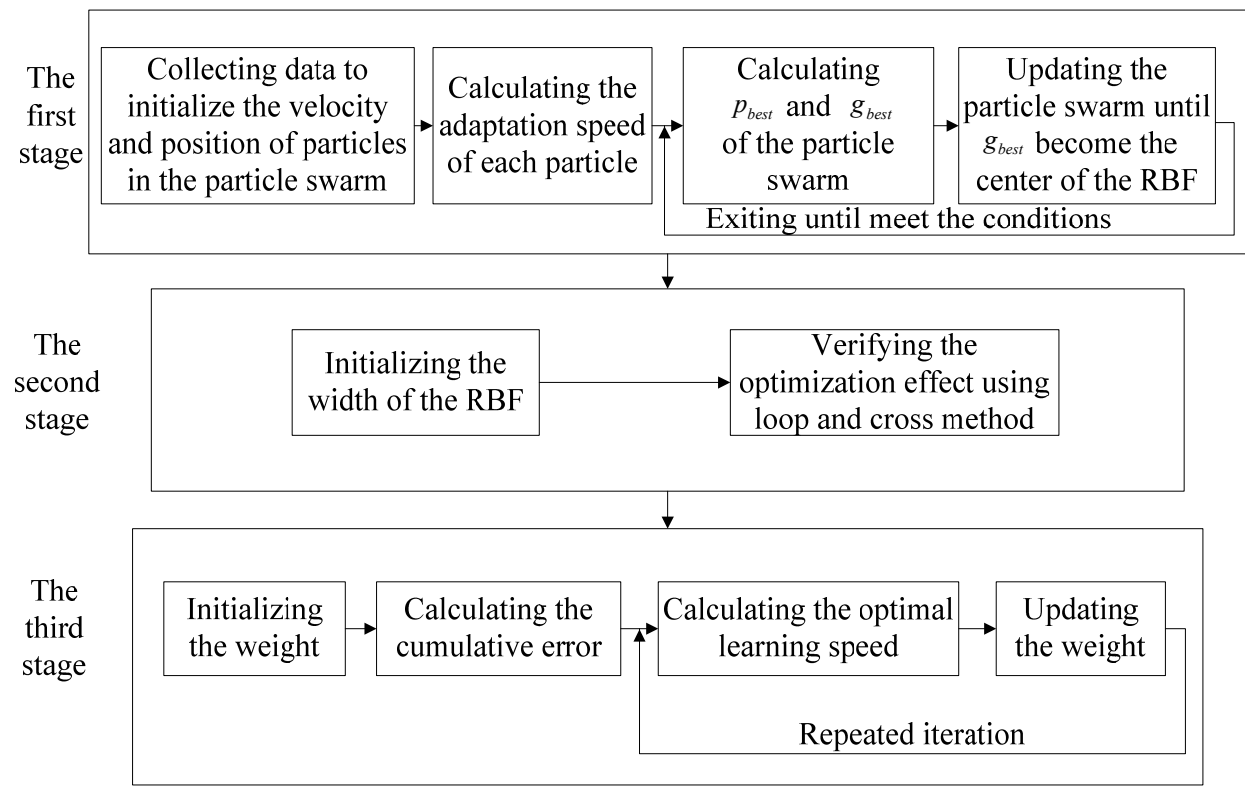




\section{Simulation and discussion}

\subsection{Output result}

\section{Test objectives}

The double lane change test is operated to obtain the longitudinal and lateral velocity, yaw rate, lateral acceleration and other test data. The yaw rate and the lateral acceleration are measured values, which can be used as inputs to the neural network.

\section{Test procedure}

The iVRU-Fx gyroscope is equipped to obtain in real-time the yaw rate and lateral acceleration of the vehicle. The LC5100 non-contact speed meter and LC-1100 space filter speed sensor are used to measure the longitudinal and lateral velocities of the vehicle. This test uses a two-contact speed sensor layout, that is to say, two speed sensors are used to obtain the longitudinal and lateral velocities in the process of tracking the double lane change test road.

The test procedure in accordance with ISO/TR3888-2004 is as follows:

Step 1: Arranging stakes shown in Figure 2 and painting prescribed path on the ground according to the double lane change test road exactly.

Step 2: Equipping the related equipments and then powering them so as to warm them up to normal operating temperature.

Step 3: With an initial velocity of $80 \mathrm{~km} / \mathrm{h}$, the tested vehicle travels along the initial lane. And then, the tested vehicle implements a lane change manoeuvre to another lane rapidly and then returns to the initial lane as soon as possible without touching any part of the stakes. At the same time, the time history curves of the measured variables are recorded.

Step 4: Repeating Step 3 process 12 times.

The measured longitudinal speed, longitudinal acceleration and yaw rate of the vehicle obtained from the double line test are shown in Figures 5-7.

Figure 5 Longitudinal speed

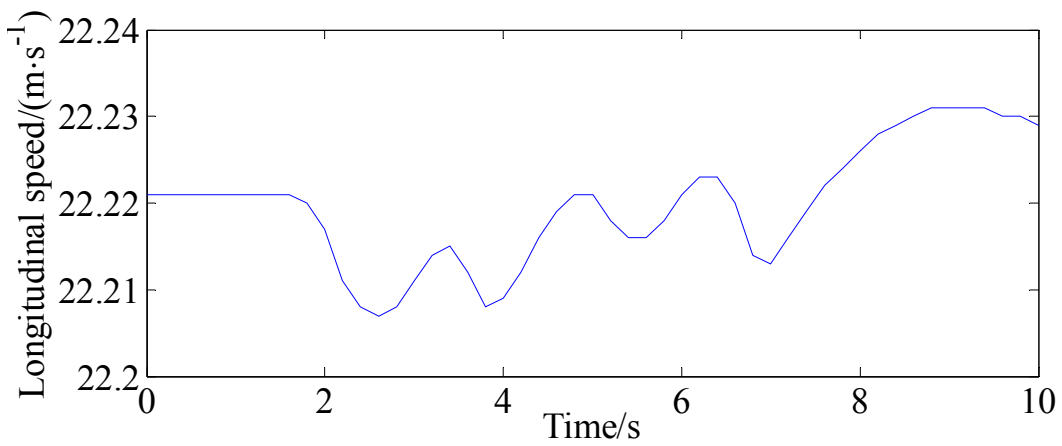


Figure 6 Longitudinal acceleration

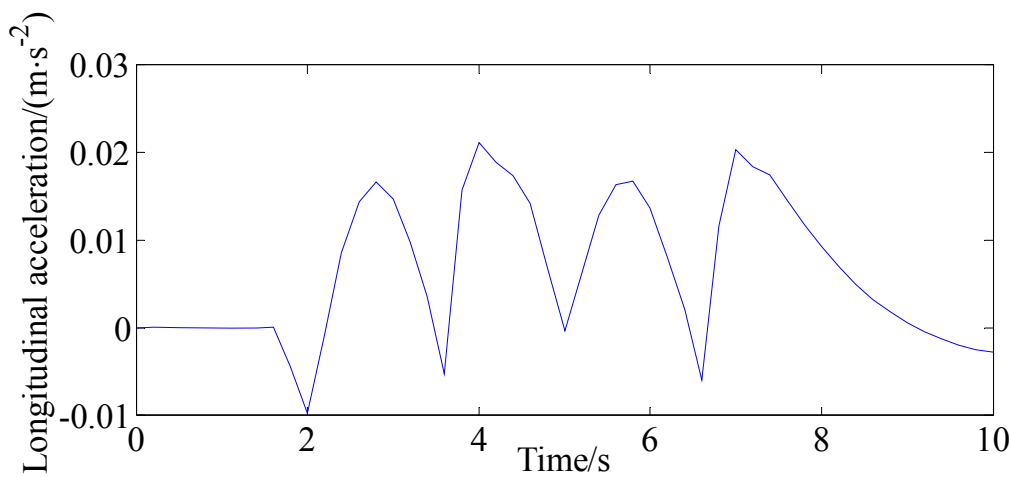

Figure 7 Yaw rate

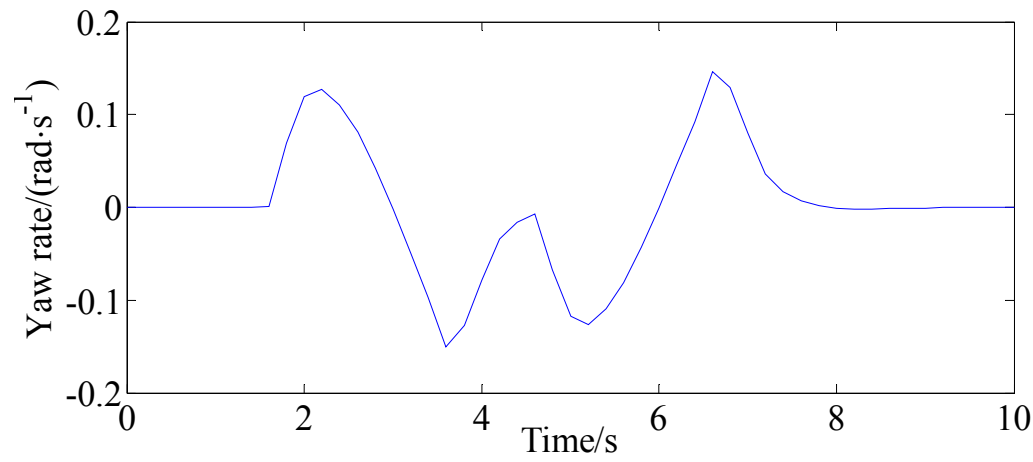

The measured signal of Figures 5-7 is input to the trained PSO-RBF network to obtain the estimation curves of the longitudinal speed, longitudinal acceleration and yaw rate, which are shown in Figures 8-10.

Figure 8 Longitudinal speed

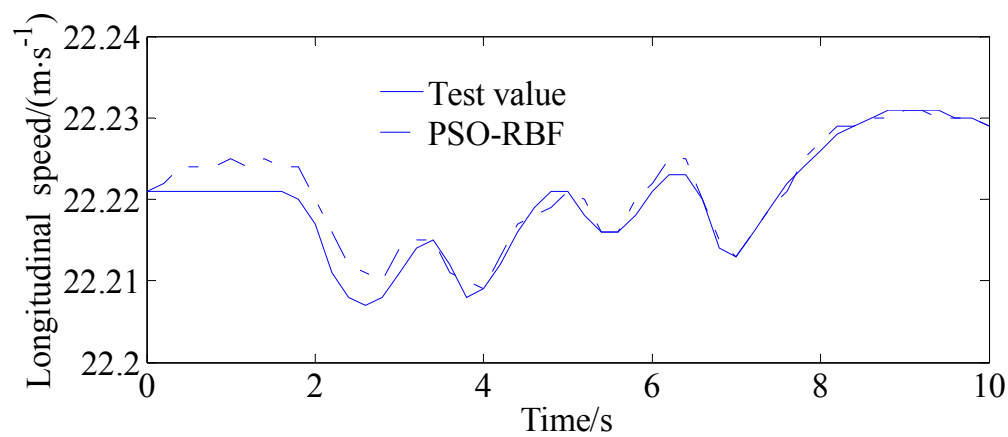


Figure 9 Longitudinal acceleration

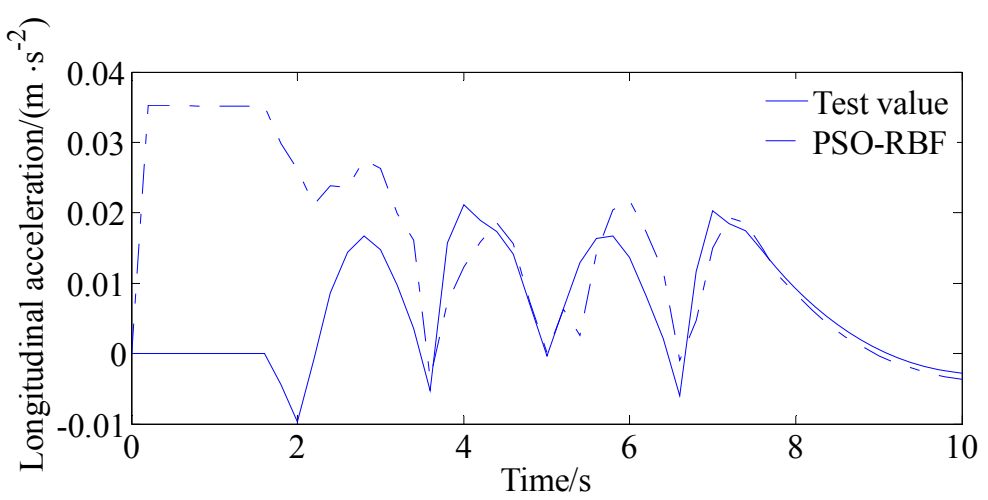

Figure 10 Yaw rate

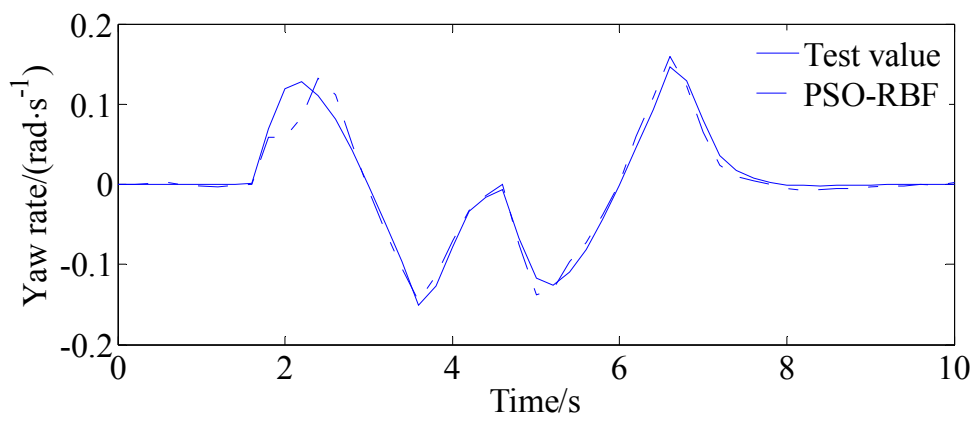

\subsection{Evaluation of calculation accuracy}

In order to verify the superiority of the PSO-RBF neural network method, the RBF neural network prediction model is used for vehicle state estimation. The simulation results are shown in Figures 11-13.

Figure 11 Longitudinal speed

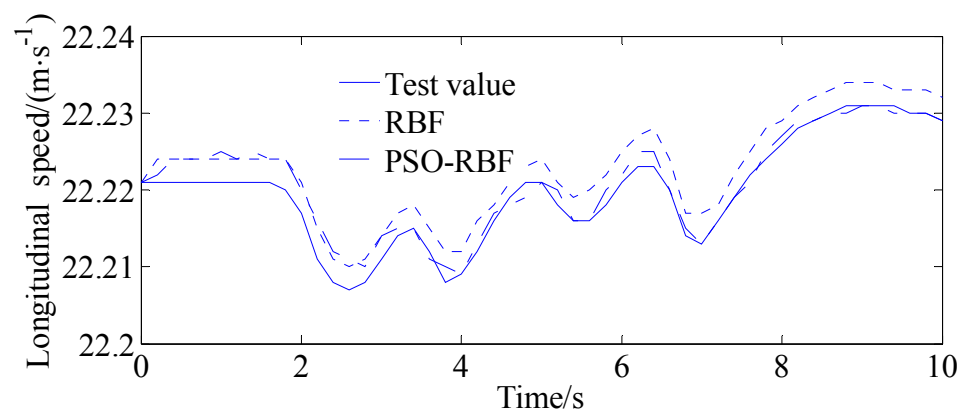


Figure 12 Longitudinal acceleration

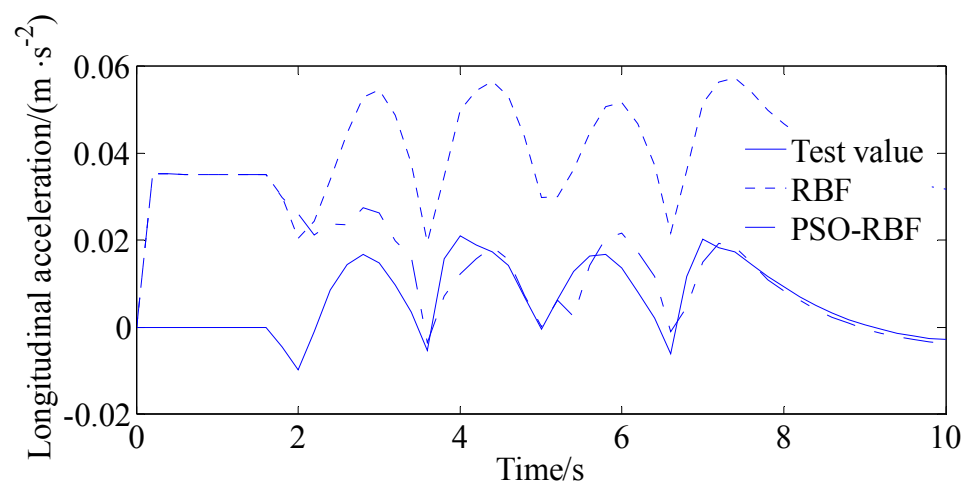

Figure 13 Yaw rate

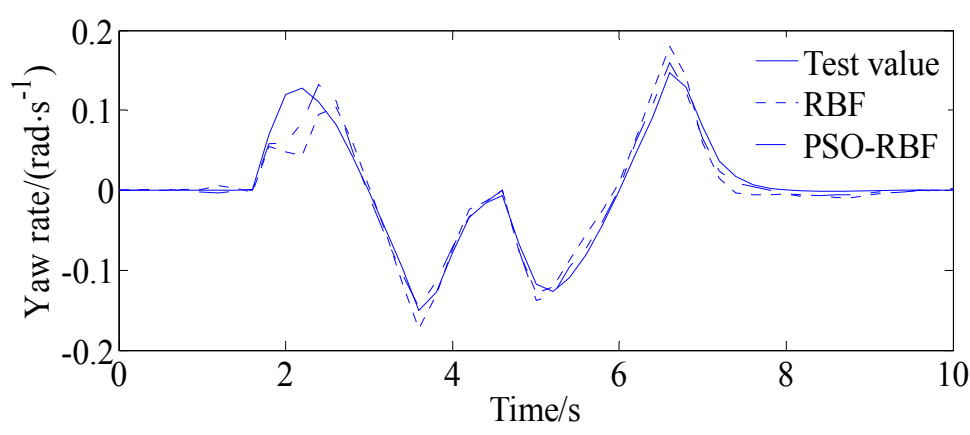

The number of iterations required for different models to achieve convergence is different. The RBF neural network needs 125 times, and the optimised PSO-RBF neural network needs 75 times. The convergence rate of the PSO-RBF network is significantly faster than the other algorithm under the same conditions indicating that the generalisation ability is stronger than the other algorithm. The convergence rate of the RBF neural network can be improved by the particle swarm optimisation.

Figure 14 shows the output error of side slip angle of the two different algorithms which are the RBF neural network and the PSO-RBF neural network.

The main reasons for the error of the side slip angle are as follows:

1 The 7-DOF vehicle model used in the estimation methods is relatively simple. The non-linear factors such as suspension is not taken into account, and the influence of damping in steering system and suspension system is neglected.

2 The test value of the side slip angle also contains a certain measurement error.

At the same time it can be seen that the RBF neural network error is between $\pm 30 \%$ and the optimised PSO-RBF neural network error is controlled in $\pm 10 \%$. The PSO-RBF neural network in the prediction accuracy of vehicle state estimation is higher than that of the RBF neural network. The PSO-RBF neural network algorithm can combine the particle swarm algorithm and the RBF neural network to make it possible to improve accuracy and computational efficiency in dealing with complex system problems. 
Figure 14 Error of side slip angle

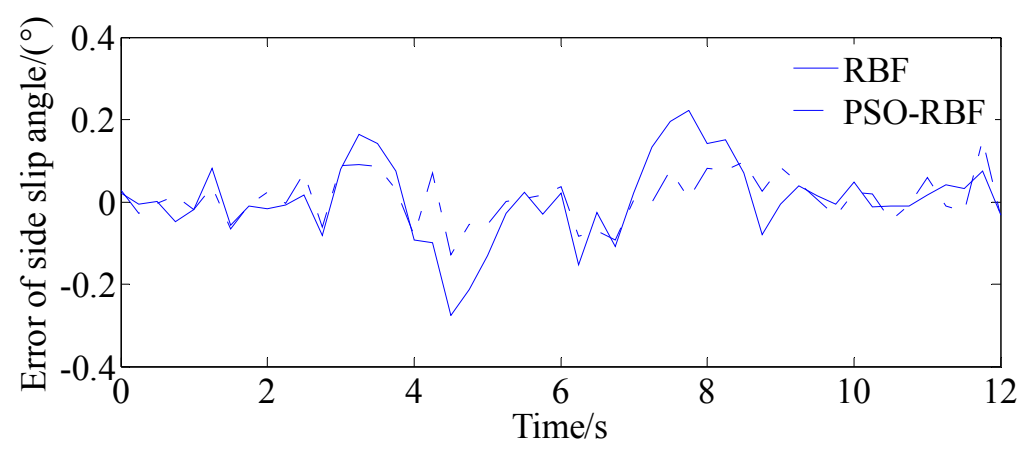

\section{Conclusions}

In this paper, the vehicle state estimation scenario is analysed while the PSO-RBF neural network method is utilised for the vehicle state estimation problem. Accordingly, a 7-DOF simplified vehicle model and the Pacejka tyre model have been used to describe the motion of the vehicle. Then the PSO-RBF neural network is operated to solve the problem of vehicle state estimation in vehicle handling dynamics.

To test the performance of the method, simulation is operated and it is shown that the vehicle states can be estimated successfully. This is a main benefit of this method for vehicle state estimation problem.

Comparison of the results of the RBF neural network method and the PSO-RBF neural network method shows that the absolute error of the side slip angle solved by the PSO-RBF neural network method is smaller than that of the RBF neural network method, indicating that the PSO-RBF neural network method can estimate the vehicle states precisely comparing with other traditional methods.

It is believed that the proposed method can considerably improve vehicle state estimation in vehicle handling dynamics.

\section{References}

Bakker, E. and Pacejka, H.B. (1989) 'A new tire model with an application in vehicle dynamics studies', SAE Paper, Vol. 98, No. 6, pp.1-13.

Bangji, Z., Haiping, D., James, L. et al. (2016) 'A novel observer design for simultaneous estimation of vehicle steering angle and sideslip angle', IEEE Transactions on Industrial Electronics, Vol. 63, No. 7, pp.4357-4366.

Bektache, D., Tolba, C. and Ghoualmi-Zine, N. (2014) 'Forecasting approach in VANET based on vehicle kinematics for road safety', International Journal of Vehicle Safety, Vol. 7, No. 2, pp.147-167.

Chen, Y.L. and Lee, W.J. (2011) 'Safety distance warning system with a novel algorithm for vehicle safety braking distance calculating', International Journal of Vehicle Safety, Vol. 5, No. 3, pp.213-231. 
Chen, Y.L., Shen, K.Y. and Wang, S.C. (2013a) 'Forward collision warning system considering both time-to-collision and safety braking distance', International Journal of Vehicle Safety, Vol. 6, No. 4, pp.347-360.

Chen, Z., Mi, C.C., Fu, Y. et al. (2013b) 'Online battery state of health estimation based on genetic algorithm for electric and hybrid vehicle applications', Journal of Power Sources, Vol. 240, pp.184-192.

Ehsan, H., Alireza, K., Saeid, K. et al. (2016) 'Estimation of longitudinal speed robust to road conditions for ground vehicles', Vehicle System Dynamics, Vol. 54, No. 8, pp.1120-1146.

Fei, S., Xuhui, H., Joachim, R. et al. (2015) 'Vehicle state estimation for anti-lock control with nonlinear observer', Control Engineering Practice, Vol. 43, pp.69-84.

Freeman, P., Wagner, J. and Alexander, K. (2016) 'Run-off-road and recovery-state estimation and vehicle control strategies', Vehicle System Dynamics, Vol. 54, No. 9, pp.1317-1343.

Funke, J. and Gerdes, J.C. (2016) 'Simple clothoid lane change trajectories for automated vehicles incorporating friction constraints', Journal of Dynamic Systems, Measurement and Control, Vol. 138, No. 2, pp.1-9.

Giulio, R., Matilde, P. and Blanco-Claraco, J-L. (2017) 'Vehicle parameter estimation using a model-based estimator', Mechanical Systems and Signal Processing, Vol. 87, pp.227-241.

Hui, Z., Guoguang, Z. and Junmin, W. (2016) 'Sideslip angle estimation of an electric ground vehicle via finite-frequency $\mathrm{H} \infty$ approach', IEEE Transactions on Transportation Electrification, Vol. 2, No. 2, pp.200-209.

Jeon, D.H. and Beak, S.M. (2011) 'Thermal modeling of cylindrical lithium-ion battery during discharge cycle', Energy Conversion and Management, Vol. 52, No. 8, pp.2973-2981.

Kiencke, U. and Nielsen, L. (2000) Automotive Control Systems, Springer, Berlin.

Lin, H.T., Liang, T.J. and Chen, S.M. (2013) 'Estimation of battery state of health using probabilistic neural network', IEEE Transactions on Industrial Informatics, Vol. 9, No. 2, pp.679-685.

Mourad, B., Abdelhamid, R., Mohammed, B. et al. (2016) 'Vehicle online parameter estimation using a nonlinear adaptive observer', 2016 American Control Conference (ACC) Boston Marriott Copley Place, Boston, MA, USA, pp.1006-1010.

Pence, B.L., Fathy, H.K. and Stein, J.L. (2014) 'Recursive estimation for reduced-order state-space models using polynomial chaos theory applied to vehicle mass estimation', IEEE Transactions on Control Systems Technology, Vol. 22, No. 1, pp.224-229.

Rajaram, V. and Subramanian, S.C. (2016) 'Design and hardware-in-loop implementation of collision avoidance algorithms for heavy commercial road vehicles', Vehicle System Dynamics, Vol. 54, No. 7, pp.871-901.

Rath, J.J., Veluvolu, K.C. and Defoort, M. (2015) 'Simultaneous estimation of road profile and tire road friction for automotive vehicle', IEEE Transactions on Vehicular Technology, Vol. 64, No. 10, pp.4461-4471.

Tan, Y.K., Mao, J.C. and Tseng, K.J. (2011) 'Modelling of battery temperature effect on electrical characteristics of li-ion battery in hybrid electric vehicle', 2011 IEEE 9th International Conference on Power Electronics and Drive Systems(PEDS), IEEE, Singapore, pp.637-642.

Wenzel, T.A., Burnham, K.J., Blundell, M.V. et al. (2007) 'Kalman filter as a virtual sensor: applied to automotive stability systems', Transactions of the Institute of Measurement and Control, Vol. 29, No. 2, pp.95-115.

Xu, L., Ching-Yao, C. and Yu, W. (2016) 'A reliable fusion methodology for simultaneous estimation of vehicle sideslip and yaw angles', IEEE Transactions on Vehicular Technology, Vol. 65, No. 6, pp.4440-4458.

Zhang, C., Li, K. and Deng, J. (2016) 'Real-time estimation of battery internal temperature based on a simplified thermoelectric model', Journal of Power Sources, Vol. 302, pp.146-154. 